\title{
Telehealth in the rehabilitation of female pelvic floor dysfunction: a systematic literature review
}

\author{
Kyannie Risame Ueda da Mata ${ }^{1}$ - Rafaela Cristina Monica Costa ${ }^{1}$ Ébe dos Santos Monteiro Carbone ${ }^{1,2}$. \\ Márcia Maria Gimenez ${ }^{1,2}$. Maria Augusta Tezelli Bortolini ${ }^{2} \cdot$ Rodrigo Aquino Castro $^{2} \cdot$ Fátima Faní Fitz $^{1,2}$ (D)
}

Received: 31 July 2020 / Accepted: 23 October 2020 / Published online: 11 November 2020

(C) The International Urogynecological Association 2020

\begin{abstract}
Introduction and hypothesis The pandemic caused by coronavirus disease 2019 (COVID-19) increased the awareness and efforts to provide care from distance using information technologies. We reviewed the literature about the practice and effectiveness of the rehabilitation of the female pelvic floor dysfunction via telehealth regarding symptomatology and quality of life and function of pelvic floor muscles (PFM).

Methods A bibliographic review was carried out in May 2020 in the databases: Embase, Medline/PubMed, LILACS and PEDro. A total of 705 articles were reviewed after the removal of duplicates. The methodological quality of the articles was evaluated by the PEDro scale. Two authors performed data extraction into a standardized spreadsheet.

Results Four studies were included, two being randomized controlled trials. Among the RCTs, only one compared telehealth with face-to-face treatment; the second one compared telehealth with postal treatment. The other two studies are follow-up and cost analysis reports on telehealth versus postal evaluation. Data showed that women who received the intervention remotely presented significant improvement in their symptoms, such as reducing the number of incontinence episodes and voiding frequency, improving PFM strength and improving quality of life compared to women who had the face-to-face treatment.

Conclusions Telehealth promoted a significant improvement in urinary symptoms, PFM function and quality of life. Telehealth is still emerging, and more studies are needed to draw more conclusions. The recommendations of the governmental authorities, physical therapy councils and corresponding associations of each country also need to be considered.
\end{abstract}

Keywords Telemedicine $\cdot$ Telemotoring $\cdot$ Pelvic floor $\cdot$ Urinary incontinence $\cdot$ Women's health

\section{Introduction}

Telehealth involves health care services, support and information provided remotely via digital communication and devices. It intends to facilitate effective delivery of health services such as physical therapy by improving access to care and information and managing health care resources [1]. Other

Supplementary Information The online version contains supplementary material available at https://doi.org/10.1007/s00192-02004588-8.

Fátima Faní Fitz

fanifitz@yahoo.com.br

Centro Universitário São Camilo, São Paulo, SP, Brazil

2 Department of Gynecology, Universidade Federal de São Paulo, Rua Napoleão de Barros, 608 - Vila Clementino, São Paulo, SP CED 04024-002, Brazil terms such as telemedicine, telemonitoring, tele-education and tele-assistance describe digital practice [2]. Due to the pandemic caused by coronavirus disease 2019 (COVID-19), health associations worldwide have released recommendations about care from distance using tools of communication and information technologies [3-5].

In clinical practice, however, it is still unclear how the professionals can perform rehabilitation of female pelvic floor dysfunction via telehealth.

Before starting a pelvic floor muscle (PFM) training program, one must ensure that the patients are able to perform a correct PFM contraction [6]. More than 30\% of women are not able to voluntarily contract the PFM at their first consultation even with individual instruction verbally and by using digital manual therapy $[7,8]$. The success rate varies from $60 \%$ to $75 \%$ when the PFM exercises are performed in the outpatient setting $[9,10]$. The literature has shown that home PFM training (PFMT) provides equal benefit to outpatient PFMT in reducing 
urinary symptoms when the patients attend some outpatient sessions to monitor their exercises during their treatment $[11$, 12]. So, the question in telehealth is: How can we perform rehabilitation of the pelvic floor in women in order to overcome the issues of not using manual therapy in the process of pelvic floor evaluation and teaching of PFM contraction?

Due to the COVID-19 pandemic, adequate patient care is urgently needed. So, alternatives in this dynamic clinical situation include systematic review as a way to connect evidence and practice and to guide clinical care [13]. Therefore, the purpose of the present study was to review telehealth in female pelvic floor dysfunction rehabilitation. The outcome of interest was the methodology by which the digital practice can be performed. Secondarily, pelvic floor symptoms, quality of life and function of the PFM were assessed.

\section{Methods}

\section{Protocol and registration}

This systematic review was developed following the PRISMA guidelines. The systematic review protocol was registered in the PROSPERO database under number CRD42020200457.

\section{Eligibility criteria and study selection}

Studies were eligible for inclusion if they were randomized controlled trials and clinical trials that used telehealth in rehabilitation of female pelvic floor dysfunction. For this review, digital practice was regarded as health care services, support and information provided remotely via digital communication as a form of intervention. We excluded studies that aimed only to investigate new technologies such as mobile applications and digital devices for home treatment with no comparator.

\section{Information sources and search}

The literature search was performed on May 2020 and included studies from inception with no language restriction. The consulted databases were: Embase, Medline/PubMed, LILACS and PEDro (Physiotherapy Evidence Database). The keywords used were: Telerehabilitation; Internet; Videoconferencing; Tele; Continence; Women's Health; Pelvic Floor; Telehealth; Urinary Incontinence; Muscle Dysfunction; Sexual Dysfunction; Pelvic Pain; Fecal Incontinence; Pelvic Organ Prolapse. The search strategies are described in the supplemental material (Supplement 1).

\section{Screening and data extraction}

A data search was performed by the authors (K.R.U.M. and R.C.M.C.). A third author (F.F.F.) was consulted for a consensus if discrepancies occurred. A standardized data extraction form was used to collect the following data: authors, year of publication, journal, country of origin, sample, age (years), objectives, outcome measure and results/conclusions. Data extraction was performed by two independent raters (E.S.M.C. and M.M.G.).

\section{Outcomes}

The primary outcome was to investigate the telehealth methodologies used by health professionals for PFM rehabilitation. Secondarily, we investigated the effectiveness of telehealth in PFM rehabilitation considering pelvic floor symptoms and quality of life as well as PFM function in women with pelvic floor dysfunctions.

\section{Risk of bias assessment and analysis}

The methodological quality of the trials was assessed using the PEDro scale (values of $0-10$ ), with scores extracted from the PEDro database [14]. The assessment of the quality of trials was performed by two independent raters (M.A.T.B. and R.A.C.), and disagreements were resolved by a third rater (F.F.F.). Methodological quality was not an inclusion criterion.

As data were extracted and described, heterogeneity between the outcomes did not allow pooling data and performing subgroup analysis or metanalysis. Results were displayed in tables in a synthesized format. The description followed a narrative review format.

\section{Results}

The first electronic database search resulted in a total of 705 articles after the removal of duplicates. As shown in Fig. 1, eight articles were selected as potentially eligible on the basis of their title and abstract, and four were excluded from analysis after reading in full [15-18]. A total of four articles were included in this review [19-21].

The methodological quality assessment by the PEDro scale revealed a median score of 5 (range 4-6) (Table 1). Random allocation, adequate follow-up, between-group comparisons and point estimates and variability were included in all trials. Concealed allocation, blind subjects, blind therapists and blind assessors were not included in all trials. Comparability at baseline was included in three trials [19, 20, 22]. Intentionto-treat analysis was reported in two publications $[19,20]$.

The articles included in this review investigated telehealth in the treatment of stress urinary incontinence (SUI) and urge urinary incontinence. Only two studies were original randomized controlled trials $[19,22]$. The other two publications were a follow-up [20] and cost analysis [21] of the study developed by Sjostrom et al. [19]. The details of the studies are described in the Table 2. 


\section{Telehealth-How is it performed?}

The studies included in this review investigated the internetbased program versus postal program [19-21] and conventional treatment (face-to-face intervention) [22].

In Sjostrom et al.'s study, the patients were recruited via an open access website. Invitations to the study were published on national websites for medical advice and as advertisements in daily newspapers. To confirm the clinical diagnosis of SUI, all participants were interviewed via telephone. The contact with patients during the intervention was asynchronous, with encrypted e-mail, requiring a separate login from both participants and therapists. The therapist gave the participant login codes for two levels at a time, with instructions to maintain training at each level for at least 1 week. The participants completed a self-evaluated test and reported a training diary to the therapist weekly. New login codes were given with the passing of every other test. The participants could contact their therapist at any time for support or questions. Response from the therapist was promised within 3 working days, and separate technical support was offered through encrypted e-mail contact with the website manager. The program was built on a secure platform, using a two-factor authentication and Secure Sockets Layer (SSL) to provide communication security over the internet. All parts of the program could be downloaded for printing [19]. In this study, the authors compared telehealth with an exercise program sent by post. The patients received a print version containing information about the program, followed by instructions for PFM training. The participants in this group had no contact with the urotherapists [19].

Hui et al. performed the PFM program via videoconferencing. The sessions were carried out in a private and quiet room in the community center. Subjects were reassured that they were only sharing the progress of their incontinence symptoms with other participants in the session. The nurse specialist provided behavioral training to the group via videoconferencing with the support of a research assistant at the patients' end. The patients shared their experiences with the nurse and were encouraged to adhere to behavioral training and PFM exercises [22].
Fig. 1 PRISMA flow diagram for the selected studies
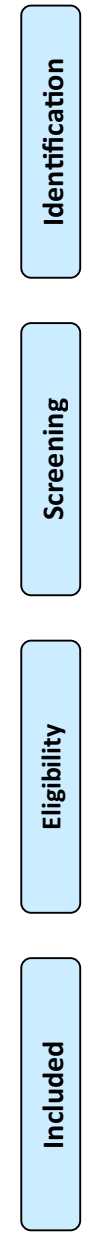

Records identified through database searching

Medline $/$ PubMed $=668$

LILACS $=17$

PEDro $=28$

Total after removing duplicates $(n=703)$

(n)

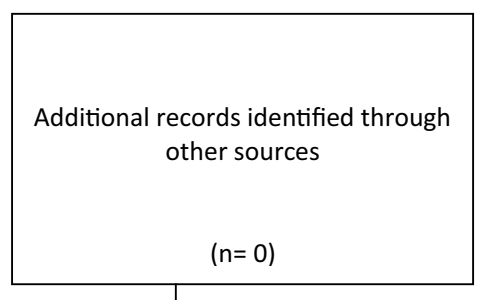

Records after duplicates removed

$(n=705)$

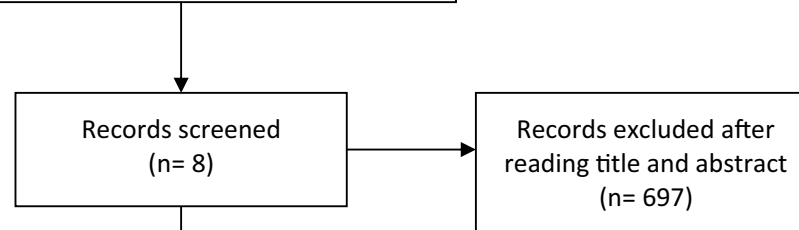

$(n=697)$

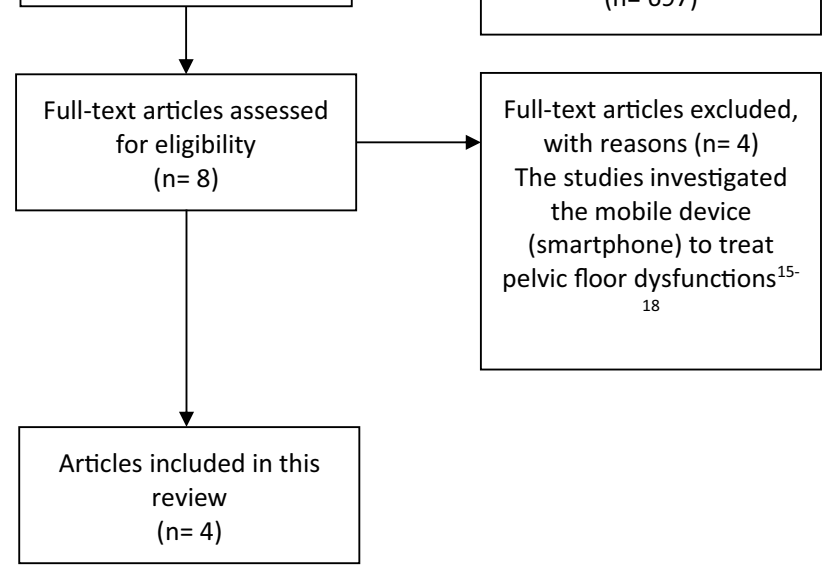




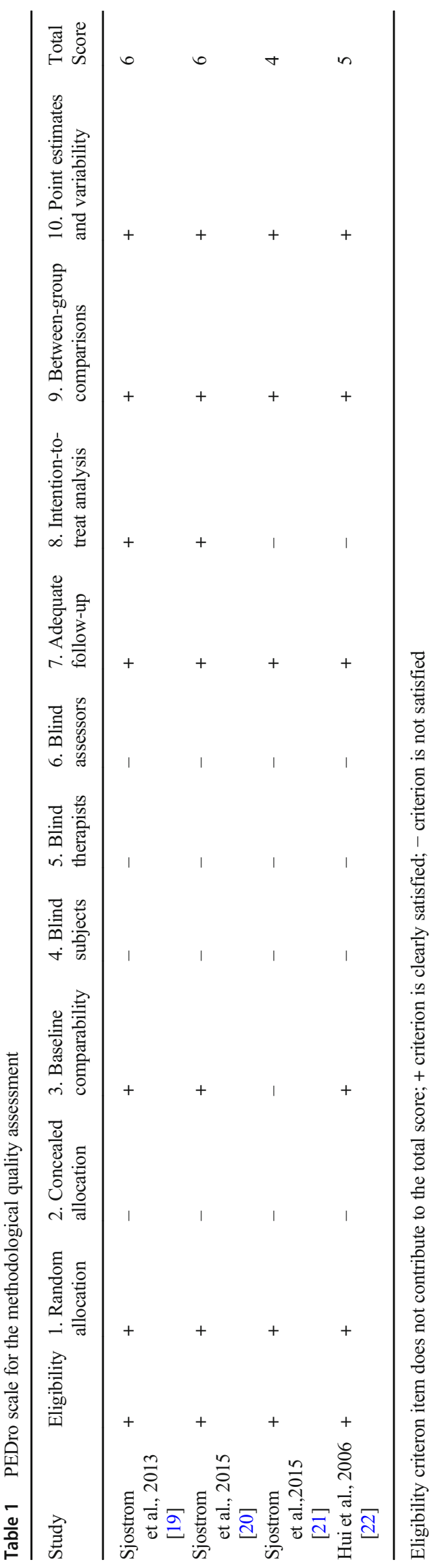
Effects of telehealth on PFM function, urinary
symptoms and quality of life

Three articles from the same Swedish group showed the findings of a program for SUI treatment via the internet versus by post on the same cohort of 250 women [19-21]. One publication presented the results on urinary symptoms, cure and quality of life after 4 months of treatment using questionnaires sent by post mail [19]; another publication reported the same outcomes at 1- and 2-year follow-up [20]; the last article addressed the costs of the two interventions [21].

Sjostrom et al. [19] described significant improvements in both interventions (internet and postal groups) after ITT analysis, but there were no significant differences between groups in urinary symptoms and condition-specific quality of life after 4-month treatment. Regarding subjective cure, more participants in the internet group reported being much or very much improved $(p=0.01)$, had reduced use of incontinence pads $(p=$ 0.02 ) and were satisfied with the treatment program $(p<0.001)$ compared to the postal group. Quality of life improved in the internet group $(p=0.001)$, but not in the postal group $(p=0.13)$. Overall, $69.8 \%(120 / 172)$ of the participants reported absence or reduced number of UI episodes by $>50 \%$ [19]. Loss to follow-up rate was $12 \%$.

The authors report $32 \%$ loss of participants at 1-year and $38 \%$ at 2 -year follow-up assessments. Highly significant $(p<0.001)$ improvements were observed for symptoms and condition-specific quality of life after 1 and 2 years, respectively, for both internet and postal interventions, without significant differences between groups. The proportions of participants perceiving they were much or very much improved were similar in both intervention groups after 1 year $(p=0.82)$, but after 2 years significantly more participants in the internet group reported this degree of improvement $(p=0.03)$. At 1 year after treatment, $69.8 \%$ of participants in the internet group and $60.5 \%$ of participants in the postal group reported that they were still satisfied with the treatment result. After 2 years, the proportions were $64.9 \%$ and $58.2 \%$, respectively [20].

The authors measured quality of life with the ICIQLUTSqol condition-specific questionnaire and calculated the quality-adjusted life-years (QALYs) gained to estimate the cost-effectiveness. Compared to the postal program, the extra cost per QALY for the internet-based program ranged from $200 €$ to $7.253 €$, indicating greater QALY gains at similar or slightly higher costs. Compared to no treatment, the extra cost per QALY for the internet-based program ranged from $10.022 €$ to $38.921 €$, indicating greater QALY gains at higher but probably acceptable costs. The authors concluded that an internet-based treatment for SUI is a new, costeffective treatment alternative [21]. 
Table 2 Details of the included randomized controlled trials

Reference Study design/ Participant characteristics, sample size Interventions period/country $(\mathrm{N})$, duration of symptoms
Outcomes (measures) and time points; results; conclusion

\begin{tabular}{ccl}
\hline $\begin{array}{c}\text { Sjostrom } \\
\text { et al., }\end{array}$ & RCT/December & Age $=18-70$ years \\
2013 & 2009 to April & $N=250$ \\
{$[19]$} & Sweden & Duration of symptoms = SUI $\geq 1$ \\
& & time/week \\
Inclusion & criteria = community-dwelling \\
& women with SUI at least once a \\
& week that matched with age. Ability \\
& to read and write Swedish and sccess \\
& to computer with internet \\
& connection \\
& & Exclusion criteria = pregnancy, \\
& previous incontinence surgery, \\
& known malignancy in lower \\
& abdomen, difficulties passing urine, \\
& macroscopic hematuria, \\
& & intermenstrual bleedings, severe \\
& psychiatric diagnosis, and \\
& neurological disease affecting \\
& sensibility in legs or lower abdomen \\
& Dropout rate = 32.4\%
\end{tabular}

Internet-based group = information on SUI and associated lifestyle factors; PFMT; training reports (frequency, time spent). This group received asynchronous, individually tailored e-mail support from a urotherapist during the treatment period

Postal group = information on SUI and Secondary outcomes = Patient Global associated lifestyle factors; PFMT; training reports (frequency, time spent). Participants in this group had no contact with the urotherapists

Follow-up: 4 months via self-assessed postal questionnaires

Primary outcomes $=$ International

Consultation on Incontinence

Questionnaire Short Form (ICIQ-UI

SF); International Consultation on Incontinence Questionnaire Lower Urinary Tract Symptoms Quality of Life (ICIQ-LUTSQoL) Impression of Improvement (PGI-I); urinary incontinence aids; patient satisfaction; EuroQol 5D-Visual Analogue Scale (EQ5D-VAS); incontinence episode frequency (IEF)

Results $=$ intention-to-treat analysis
Sjostrom

et al.,

2015

[20]
Follow-up was performed after 1 and 2 years via self-assessed postal questionnaires

There was no face-to-face contact with the participants at any time showed high significance with both interventions, but there were no significant differences between groups in primary outcomes. $40.9 \%$ of internet group perceived they were much or very much improved; $59.5 \%$ reported reduced usage of incontinence aids; $84.8 \%$ were satisfied with the treatment program vs. $26.5 \% ; 41.4 \%$ and $62.9 \%$, respectively, of postal group

Conclusion $=$ Concerning primary outcomes, treatment effects were similar between groups whereas for secondary outcomes the internet-based treatment was more effective. Internet-based treatment for SUI is a new, promising treatment alternative

Results $=$ Within both treatment groups, there were highly significant improvements in the primary outcomes, ICIQ-UI SF and ICIQ-LUTSqol, after 1 and 2 years compared with the baseline. The difference between the groups was not significant after 1 year. After 2 years, significantly more participants in the internet group rated their leakage as much or very much improved than was the case in the postal group. Health-specific QoL did not improve significantly in any of the treatment groups after 1 year. However, after 2 years there was significant improvement within the internet group, but not within the postal group. The differences between the groups were not significant

Conclusion $=$ Non-face-to-face treatment of SUI with PFMT provides significant and clinically relevant improvements in symptoms and condition-specific QoL at 1 and 2 years after treatment 
Table 2 (continued)

Reference Study design/ Participant characteristics, sample size Interventions period/country $(\mathrm{N})$, duration of symptoms
Outcomes (measures) and time points; results; conclusion

Sjostrom
et al.,
2015
$[21]$

Hui et al., RCT/not 2006 reported/ [22] China
Age $=60$ years or over
$N=58$

\section{Inclusion}

criteria $=$ community-dwelling older women aged 60 years or over, with symptoms of urge or SUI, and with one or more incontinence episodes in a week

Exclusion criteria $=$ active urinary tract infection, a post-void residual volume by bladder ultrasound $>$ $150 \mathrm{ml}$, third-degree uterine prolapse and those already receiving treatment for their urinary symptoms
Included all relevant costs accrued during the first year, regardless of who paid for them. Prices per unit were multiplied by the amount consumed and added up to a sum representing the total societal cost. All costs are given in euros at the 2010 mid-year level

Follow-up $=1$ year
Outcomes $=$ incremental cost effectiveness ratio (ICER); International Consultation on Incontinence Questionnaire Short Form (ICIQ-UI SF); International Consultation on Incontinence Questionnaire Lower Urinary Tract Symptoms Quality of Life (ICIQ-LUTSQoL); quality-adjusted life-years (QALYs).

Results = compared to the postal program, the extra cost per QALY for the internet-based program ranged from $200 €$ to $7253 €$, indicating greater QALY gains at similar or slightly higher costs.

Compared to no treatment, the extra cost per QALY for the internet-based program ranged from $10,022 €$ to $38,921 €$, indicating greater QALY gains at higher, but probably acceptable costs

Conclusion $=$ an internet-based treatment for SUI is a new, cost-effective treatment alternative

Outcomes $=$ perception of the severity of incontinence symptoms and level of satisfaction $(0=$ none, $1=$ mild, 2 = moderate, $3=$ severe); 3 -day voiding diary (number of incontinent episodes, voiding frequency and voided volume); pelvic floor muscle strength by digital assessment [Oxford Scale $(0=$ none, $1=$ flicker, 2 = weak, 3 = moderate, $4=$ good, $5=$ strong)]; satisfaction with the TCP on a 6-point Likert scale ( $0=$ highly dissatisfied to $5=$ highly satisfied).

Results = participants in both treatment groups experienced significant improvement in their symptoms with a reduction in the number of daily incontinence episodes and voiding frequency, while the volume of urine at each micturition increased. Pelvic floor muscle strength also improved. There were no significant differences in outcomes between the two groups

Conclusion $=$ results suggested that videoconferencing is as effective as conventional methods in the management of urinary incontinence

PFMT pelvic floor muscle training, SUI stress urinary incontinence, ICIQ-UI SF International Consultation on Incontinence Questionnaire Short Form, ICIQ-LUTSqol International Consultation on Incontinence Questionnaire Lower Urinary Tract Symptoms Quality of Life, PGI-I Patient Global Impression of Improvement, $E Q 5 D$-VAS EuroQol 5D-Visual Analogue Scale, IEF incontinence episode frequency, ICER incremental cost effectiveness ratio, $Q A L Y S$ quality-adjusted life-years, TCP telemedicine continence group, $C S$ continence service 
Hui et al. compared telemedicine with conventional outpatient sessions (face to face) in 58 community-dwelling older women with urgency or SUI. There were no significant differences in outcomes between the two groups. Participants in both treatment groups experienced significant improvement in their symptoms, namely, reduction in the number of daily incontinence episodes $(p<0.001)$ and voiding frequency ( $\mathrm{p}$ $<0.001$ ), while the volume of urine at each micturition increased $(p<0.005)$. PFM strength as measured by the Oxford Scale also improved $(p<0.005)$ [22].

\section{Discussion}

Telehealth is an alternative way to provide rehabilitation services. Technological advances prove to be a facilitator in the communication between the health professional and the patient especially in remote locations, presenting great potential as a substitute or as a complement to current therapies [23]. Digital practice is not a modality used in all countries and depends on the local regulations.

The main question is how to use digital technology in the clinical practice of PFM rehabilitation. In this review we gathered data from two controlled trials that addressed teleconsultation and telemonitoring in the rehabilitation of PFM for the treatment of urinary disorders [19-22].

Sjostrom et al. used a PFMT program via the internet compared to a postal program. For the authors a standardized faceto-face treatment or care as usual would have been an option to compare the internet modality, but they wanted the treatment program to be accessible to women from all over the country, even from remote areas or from areas with inadequate staffing [19]. Both modalities were shown to be beneficial treatments after 4-month intervention [19] and at 1 and 2 years after treatment [20]; thus, management of SUI without faceto-face contact is possible and may increase access to care. The authors also found the internet-based treatment is an effective new, promising, alternative treatment $[19,20]$.

Before starting a PFM training program, one has to ensure that the patients are able to perform a correct PFM contraction [6]. In the study developed by Sjostrom et al., all the interventions were made without PFM evaluation. The authors did not explain how the patients were directed to perform the PFM contraction, but they acknowledged that the ability to understand written instructions, carry them out and adequately use a computer were prerequisites to succeed with a treatment completed on one's own in the internet group [19].

On the other hand, Hui et al. assessed the PFM strength face to face with biofeedback (BF) and vaginal palpation at baseline in both groups (telemedicine continence group and conventional group) in their study [22]. The literature suggests the utilization of sensor technologies to sample and quantify movement in telehealth [24]. BF equipment uses sensors to measure PFM contraction and maybe can be used in telehealth. There is no consensus in the literature on whether $\mathrm{BF}$ can be used to promote awareness of the PFM. Some authors have tried to make patients more aware of muscle function [25]. To date, there are no studies investigating the effect of $\mathrm{BF}$ in a population that is not able to contract the PFM. In others' opinions, it is difficult to understand how the $\mathrm{BF}$ machine can teach the patient how to contract the PFM without verbal instruction and manual techniques explained by the therapist [26].

Conservative treatment depends heavily on physical touch, and therapists rely on the objective measurement of physical performance to inform diagnosis and intervention [24]. With this, therapies that do not typically involve hands-on assessment are best suited to digital practice. Even though in PFM rehabilitation it is difficult to adapt the practice to provide services via information and communication technologies, as hands-on assessment and treatment are typically involved, it seems that it is feasible to teach the correct PFM contraction technique with verbal instructions and drawings of the anatomy of the pelvic floor and to help the patients understand the action of the PFM, describing the contraction as a lift starting with closure of the doors (squeeze) and from there the elevator is moving upstairs (lift) [6].

The virtual environment can be displayed to the patient via computer screen, or fully immersive environments are possible with the use of head-mounted visual displays and feedback devices [24]. Sjostron et al. used a program developed on a secure platform with two-factor authentication [19]. Hui et al. used videoconferencing, and participants shared their experiences with the group members. The only request was that the patients remain in a private and silent room [22]. A private environment, with the use of headphones so that other professionals and/or family members do not have access to the session, should be recommended. The risk of leakage of information related to digital care always exists and must be shared with patients, and, whenever possible, safer professional platforms should be used [27].

The patient's knowledge and ability related to the virtual environment must be considered. Some patients considered the double logins complicated in the study of Sjostrom et al. [19]. Therefore, it is recommended that service providers ensure that technical requirements are met, provide access to technical support and provide training to all users [23]. The lack of ability and knowledge related to handling technologies are the disadvantages of telerehabilitation [28].

The literature reports that the savings of transportation costs and of the health care system's and patient's time, the continuity of patient care that can be achieved through the remote provision and the heightened ability to control the timing, intensity and sequencing of the intervention are advantages of telerehabilitation [24]. The questions are whether the professionals are prepared to implement digital practice and 
how they can offer telerehabilitation services, especially in the area of pelvic floor dysfunction rehabilitation. This type of service is a growing field, adopted within the COVID-19 pandemic, and has the potential to reduce costs, increase the overall accessibility of modern health care systems and open new perspectives for rehabilitation in pelvic floor dysfunctions $[2$, 29]. A guide with specific issues involving digital practice in physical therapy needs to developed. And the governmental authorities, physical therapy councils and corresponding associations of each country should be involved.

The limitations of this study include the scarcity of literature related to telehealth, especially compared to conventional treatment (face to face). The study performed by Hui et al. was the only one that compared telehealth with conventional treatment. The authors concluded that telehealth is as effective as conventional treatment for urinary incontinence [22]. The literature reports on telehealth and conventional treatment in other fields of medicine that require conservative therapy. As with all fields and interventions, effective telehealth requires that therapists understand the essential components of their treatments and ensure that they are carefully included in care $[28,29]$. Thus, because of the specificity of each field, it is not possible to discuss the results found by Hui et al. with further scientific depth. Another limitation is the fact that the review was carried out in a short period, limiting the search in the gray literature, and the article was produced quickly so that it could be useful to professionals and patients.

\section{Conclusion}

The literature on digital practice in the treatment of pelvic floor dysfunction is scarce. Only two original studies have investigated telehealth in SUI treatment, and one of them was without a face-to-face group. Telehealth promoted a significant improvement in urinary symptoms, PFM function and quality of life. The results of the studies showed that internetbased treatment is a promising treatment alternative. However, this type of assistance still needs to be studied to verify its real benefits in the treatment of pelvic floor dysfunctions, since further clarification is needed on how to perform it. The recommendations of governmental authorities, physical therapy councils and corresponding associations of each country also need to be considered.

\section{Compliance with ethical standards}

Conflict of interest The authors declare no conflicts of interest.

Abbreviations COVID-19, Coronavirus Disease 2019; PFM, Pelvic floor muscle; PFMT, Pelvic floor muscle training; PEDro, Physiotherapy Evidence Database; SUI, Stress urinary incontinence

\section{Appendix}

PEDro: Telerehabiltation AND Continence and women's health; Tele AND Continence and women's health; Video conferecing AND Continence and women's health; Internet AND Continence and women's health.

LILACS: Telerehabilitation AND Pelvic Floor; Telerehabilitation AND Telehealth AND Women's Health; Videoconferencing AND Pelvic Floor; videoconferencing AND Urinary Incontinence (tw:(Pelvic Prolapse)) AND (tw:(telerehabilitation)); (tw:(telerehabilitation)) AND (tw:(telehealth)) AND (tw:(women's health)) AND (tw:(Pelvic Prolapse)); (tw:(women's health)) AND (tw:(Pelvic Prolapse)) AND (tw:(Video Virtual)); (tw:(Videoconferencing)) AND (tw:(Pelvic Prolapse)); (tw:(Videoconferencing)) AND (tw:(fecal incontinence)); (tw:(Video Virtual)) AND (tw:(fecal incontinence)); (tw:(telerehabilitation)) AND (tw:(women's health)) AND (tw:(fecal incontinence)).

PubMed: ((Internet) AND Continence) AND Women's Health; (Tele) AND Pelvic Floor; (Internet) AND Pelvic Floor; (((Telerehabilitations) OR (Tele-rehabilitation) OR (Tele rehabilitation) OR (Tele-rehabilitations) OR (Remote Rehabilitation) OR (Rehabilitation, Remote) OR (Rehabilitations, Remote) OR (Remote Rehabilitations) OR (Virtual Rehabilitation) OR (Rehabilitation, Virtual) OR (Rehabilitations, Virtual) OR (Virtual Rehabilitations) OR)) AND Pelvic Floor; (Video Conferencing) AND Pelvic Floor; (Video Conferencing) AND Pelvic Floor; $((((($ Telehealth) AND Women's Health)) AND ((Telerehabilitation) OR (Tele-rehabilitation) OR (Tele rehabilitation) OR (Tele-rehabilitations) OR (Remote rehabilitation) OR (Rehabilitation, remote) OR (Rehabilitations, remote) OR (Remote rehabilitations) OR (Virtual rehabilitation) OR (Rehabilitation, virtual) OR (Rehabilitations, virtual) OR (Virtual rehabilitations)))) AND ((Video conferencing) AND Pelvic floor muscle dysfunction); (((((Telehealth) AND Women's health)) AND ((telerehabilitation) OR (telerehabilitation) OR (tele rehabilitation) OR (tele-rehabilitations) OR (remote rehabilitation) OR (rehabilitation, remote) OR (rehabilitations, remote) OR (remote rehabilitations) OR (virtual rehabilitation) OR (rehabilitation, virtual) OR (rehabilitations, virtual) OR (virtual rehabilitations)))) AND ((video conferencing) AND pelvic floor muscle dysfunction); (((telehealth) AND women's health)) AND ((telerehabilitation) OR (tele-rehabilitation) OR (tele rehabilitation) OR (tele-rehabilitations) OR (remote rehabilitation) OR (rehabilitation, remote) OR (rehabilitations, remote) OR (remote rehabilitations) OR (virtual rehabilitation) OR (rehabilitation, virtual) OR (rehabilitations, virtual) OR (virtual rehabilitations)); (telehealth) AND women's health; ((c((telerehabilitation) OR (tele-rehabilitation) OR (tele rehabilitation) OR (tele-rehabilitations) OR (remote rehabilitation) OR (rehabilitation, remote) OR (rehabilitations, remote) OR (remote rehabilitations) OR (virtual rehabilitation) OR (rehabilitation, 
virtual) $\mathrm{OR}$ (rehabilitations, virtual) $\mathrm{OR}$ (virtual rehabilitations))) AND Women's health)) AND telehealth; (video conferencing) AND pelvic floor muscle dysfunction; (video conferencing) AND pelvic floor muscle dysfunction; ((((Telerehabilitations) OR (Tele-rehabilitation) OR (Tele rehabilitation) OR (Telerehabilitations)OR (Remote Rehabilitation) OR (Rehabilitation, Remote) OR (Rehabilitations, Remote) OR (Remote Rehabilitations) OR (Virtual Rehabilitation) OR (Rehabilitation, Virtual)OR (Rehabilitations, Virtual) OR (Virtual Rehabilitations) OR)) AND video conferencing) AND ((((telerehabilitation) OR (tele-rehabilitation) OR (tele rehabilitation) OR (tele-rehabilitations) OR (remote rehabilitation) OR (rehabilitation, remote) OR (rehabilitations, remote) OR (remote rehabilitations) OR (virtual rehabilitation) OR (rehabilitation, virtual) OR (rehabilitations, virtual) OR (virtual rehabilitations))) AND pelvic floor muscle dysfunction); ((((Telerehabilitations) OR (Tele-rehabilitation) OR (Tele rehabilitation) OR (Telerehabilitations)OR (Remote Rehabilitation) OR (Rehabilitation, Remote) OR (Rehabilitations, Remote) OR (Remote Rehabilitations) OR (Virtual Rehabilitation) OR (Rehabilitation, Virtual)OR (Rehabilitations, Virtual) OR (Virtual Rehabilitations) OR)) AND video conferencing) AND ((((telerehabilitation) OR (tele-rehabilitation) OR (tele rehabilitation) OR (tele-rehabilitations) OR (remote rehabilitation) OR (rehabilitation, remote) OR (rehabilitations, remote) OR (remote rehabilitations) OR (virtual rehabilitation) OR (rehabilitation, virtual) OR (rehabilitations, virtual) OR (virtual rehabilitations))) AND pelvic floor muscle dysfunction); (((telerehabilitation) OR (tele-rehabilitation) OR (tele rehabilitation) OR (telerehabilitations) OR (remote rehabilitation) OR (rehabilitation, remote) OR (rehabilitations, remote) OR (remote rehabilitations) OR (virtual rehabilitation) OR (rehabilitation, virtual) OR (rehabilitations, virtual) OR (virtual rehabilitations))) AND pelvic floor muscle dysfunction; (((telerehabilitation) OR (telerehabilitation) OR (tele rehabilitation) OR (tele-rehabilitations) OR (remote rehabilitation) OR (rehabilitation, remote) OR (rehabilitations, remote) OR (remote rehabilitations) OR (virtual rehabilitation) OR (rehabilitation, virtual) OR (rehabilitations, virtual) OR (virtual rehabilitations))) AND sexual dysfunction; (((telerehabilitation) OR (tele-rehabilitation) OR (tele rehabilitation) OR (tele-rehabilitations) OR (remote rehabilitation) OR (rehabilitation, remote) OR (rehabilitations, remote) OR (remote rehabilitations) OR (virtual rehabilitation) OR (rehabilitation, virtual) OR (rehabilitations, virtual) OR (virtual rehabilitations))) AND pelvic pain; (((telerehabilitation) OR (tele-rehabilitation) OR (tele rehabilitation) OR (tele-rehabilitations) OR (remote rehabilitation) OR (rehabilitation, remote) OR (rehabilitations, remote) OR (remote rehabilitations) OR (virtual rehabilitation) OR (rehabilitation, virtual) OR (rehabilitations, virtual) OR (virtual rehabilitations))) AND urinary incontinence; (((telerehabilitation) OR (tele-rehabilitation) OR (tele rehabilitation) OR (telerehabilitations) OR (remote rehabilitation) OR (rehabilitation, remote) OR (rehabilitations, remote) OR (remote rehabilitations)
OR (virtual rehabilitation) OR (rehabilitation, virtual) OR (rehabilitations, virtual) OR (virtual rehabilitations))) AND Women's health; telerehabilitation) OR (tele-rehabilitation) OR (tele rehabilitation) OR (tele-rehabilitations) OR (remote rehabilitation) OR (rehabilitation, remote) OR (rehabilitations, remote) OR (remote rehabilitations) OR (virtual rehabilitation) OR (rehabilitation, virtual) OR (rehabilitations, virtual) OR (virtual rehabilitations) Telerehabilitation[MeSH Terms]Pelvic Prolapse; $(((()(($ Telehealth $)$ AND Women's health $))$ AND ((telerehabilitation) OR (tele-rehabilitation) OR (tele rehabilitation) OR (tele-rehabilitations) OR (remote rehabilitation) OR (rehabilitation, remote) OR (rehabilitations, remote) OR (remote rehabilitations) OR (virtual rehabilitation) OR (rehabilitation, virtual) OR (rehabilitations, virtual) OR (virtual rehabilitations)))) AND ((video conferencing) AND pelvic floor muscle dysfunction))) AND (Pelvic Prolapse[MeSH Terms]); (((Pelvic Prolapse) AND (Tele)) AND (Telerehabilitation[MeSH Terms])) AND (Internet[MeSH Terms]); (telehealth) AND women's health; ((c((telerehabilitation) OR (tele-rehabilitation) OR (tele rehabilitation) OR (tele-rehabilitations) OR (remote rehabilitation) OR (rehabilitation, remote) OR (rehabilitations, remote) OR (remote rehabilitations) OR (virtual rehabilitation) OR (rehabilitation, virtual) OR (rehabilitations, virtual) OR (virtual rehabilitations))) AND Women's health)) AND telehealth; (video conferencing) AND pelvic floor muscle dysfunction AND Pelvic Prolapse; (((Telerehabilitations) OR (Tele-rehabilitation) OR (Tele rehabilitation) OR (Tele-rehabilitations)OR (Remote Rehabilitation) OR (Rehabilitation, Remote) OR (Rehabilitations, Remote) OR (Remote Rehabilitations) OR (Virtual Rehabilitation) OR (Rehabilitation, Virtual)OR (Rehabilitations, Virtual) OR (Virtual Rehabilitations) OR)) AND Pelvic Floor AND Pelvic Prolapse; Telerehabilitation AND Internet AND Fecal Incontinence; Fecal incontinences AND Tele; (((Telerehabilitations) OR (Tele-rehabilitation) OR (Tele rehabilitation) OR (Tele-rehabilitations)OR (Remote Rehabilitation) OR (Rehabilitation, Remote) OR (Rehabilitations, Remote) OR (Remote Rehabilitations) OR (Virtual Rehabilitation) OR (Rehabilitation, Virtual)OR (Rehabilitations, Virtual) OR (Virtual Rehabilitations) OR)) AND Pelvic Floor AND tele AND fecal incontinence; Women's Health AND Videoconferencing AND fecal Incontinence; Fecal Incontinence AND internet.

\section{EMBASE.}

(Physioterapy OR Telerehabilitation) AND Women'S AND Health AND 'Sexual Dysfunction'; (Physio AND Terapy OR Telerehabilitation)AND Women'S AND Health AND 'Sexual Dysfunction'; (Physio AND Terapy OR Telerehabilitation) AND Women'S AND Health AND Urinary AND Incontinence; (Telerehabilitation OR Tele) AND Women's AND Health AND Urinary AND Incontinence; Physical AND Therapy AND Telerehabilitation AND Women'S AND Health AND Urinary AND Incontinence; Telerehabilitation AND Internet 
AND Urinary AND Incontinence AND 'Women'S Health'; Telerehabilitation AND Tele AND 'Urine Incontinence' AND 'Women'S Health'; Telerehabilitation AND Tele AND 'Pelvic Floor' AND 'Women'S Health'; Telerehabilitation AND Tele AND Pelvic AND Organ AND Prolapse AND 'Women'S Health'; Telerehabilitation AND Tele AND Incontinence AND Urinary; ('Telerehabilitation'/ Exp OR Telerehabilitation) AND Tele AND Continence; ('Videoconferencing'/Exp OR Videoconferencing) AND Tele AND Pelvic AND Floor; Tele AND Sexual AND Dysfunction AND Videoconferencing; ('Telerehabilitation'/ Exp OR Telerehabilitation) AND Sexual AND Dysfunction AND Videoconferencing; ('Telehealth'/Exp OR Telehealth OR Telerehabilitation:Ti) AND 'Womens Health' AND 'Pelvic Floor'; ('Videoconferencing'/Exp OR Videoconferencing) AND 'Womens Health' AND 'Pelvic Floor'; ('Videoconferencing'/Exp OR Videoconferencing) AND 'Womens Health' AND 'Pelvic Floor'; Telerehabilitation AND 'Muscle Dysfunction' AND Tele; Telerehabilitation AND Fecal AND Incontinence AND Tele AND 'Women'S Health'; Telerehabilitation AND Tele AND 'Women'S Health' AND Pelvic AND Pain; Telerehabilitation AND Telehealth AND 'Women'S Health' AND Pelvic AND Pain; ('Videoconferencing'/Exp OR Videoconferencing) AND Telerehabilitation AND 'Womens Health' AND 'Pelvic Floor'; ('Internet'/Exp OR Internet) AND Telerehabilitation AND 'Womens Health' AND 'Pelvic Floor'; ('Telerehabilitation'/Exp OR Telerehabilitation) AND Telerehabilitation AND 'Womens Health' AND 'Pelvic Floor'; ('Telerehabilitation'/Exp OR Telerehabilitation) AND Telerehabilitation AND 'Womens Health' AND 'Pelvic Floor'; ('Telerehabilitation'/Exp OR Telerehabilitation) AND 'Womens Health'; ('Telerehabilitation'/Exp OR Telerehabilitation) AND 'Pelvic Floor'; ('Pelvic Floor'/Exp OR 'Pelvic Floor' OR (('Pelvic'/Exp OR Pelvic) AND Floor)) AND Telerehabilitation

\section{References}

1. Digital Practice White Paper and Survey. http://www.inptra.org/ Resources/DigitalPracticeWhitePaperandSurvey.aspx. Accessed 30 April, 2020.

2. Dantas LO, Barreto RPG, Ferreira CHJ. Digital physical therapy in the COVID-19 pandemic [published online ahead of print, 2020 May 1]. Braz J Phys Ther. 2020;S1413-3555(20):30402-0. https://doi.org/10.1016/j.bjpt.2020.04.006.

3. Chartered Society of Physiotherapy. Remote service delivery options. https://www.csp.org.uk/news/coronavirus/remote-servicedelivery-options. Accessed 30 April, 2020.

4. Canadian Physiotherapy Association. Tele-Rehabilitation. https:// physiotherapy.ca/telerehabilitation. Accessed 30 April, 2020.
5. Conselho Federal de Fisioterapia e Terapia Ocupacional (COFFITO). Resolução No 516, de 20 de março de 2020 Teleconsulta, Telemonitoramento e teleconsultoria; 2020. https:// www.coffito.gov.br/nsite/?p=15825. Accessed 30 April, 2020.

6. Bø K, Mørkved S. Ability to contract the pelvic floor muscles. In: Bø K, Berghmans B, Mørkved S, Van Kampen M, editors. Evidence-based physical therapy for the pelvic floor. Edinburgh: Elsevier; 2015. p. 110-7.

7. Kegel AH. Stress incontinence and genital relaxation; a nonsurgical method of increasing the tone of sphincters and their supporting structures. Ciba Clin Symp. 1952;4(2):35-51.

8. Bump R, Hurt WG, Fantl JA, Wyman JF. Assessment of Kegel exercise performance after brief verbal instruction. Am J Obstet Gynecol. 1991;165:322-9. https://doi.org/10.1016/0002-9378(91) 90085-6.

9. Castro RA, Arruda RM, Zanetti MR, Santos PD, Sartori MG, Girão MJ. Single-blind, randomized, controlled trial of pelvic floor muscle training, electrical stimulation, vaginal cones, and no active treatment in the management of stress urinary incontinence. Clinics (Sao Paulo). 2008;63(4):465-72. https://doi.org/10.1590/ s1807-59322008000400009.

10. Bø K, Hagen RH, Kvarstein B, Jørgensen J, Larsen S. Pelvic floor muscle exercise for the treatment of female urinary stress incontinence: III. Effects of two different degrees of pelvic floor muscle exercises. Neurourol Urodyn. 1990;9:489-502. https://doi.org/10. 1002/nau.1930090505.

11. Fitz FF, Gimenez MM, de Azevedo Ferreira L, Matias MMP, Bortolini MAT, Castro RA. Pelvic floor muscle training for female stress urinary incontinence: a randomised control trial comparing home and outpatient training. Int Urogynecol J. 2020;31(5):98998. https://doi.org/10.1007/s00192-019-04081-x.

12. Felicíssimo MF, Carneiro MM, Saleme CS, Pinto RZ, da Fonseca AM, da Silva-Filho AL. Intensive supervised versus unsupervised pelvic floor muscle training for the treatment of stress urinary incontinence: a randomized comparative trial. Int Urogynecol J. 2010;21(7):835-40. https://doi.org/10.1007/s00192-010-1125-1.

13. Bagg MK, McAuley JH. Correspondence: living systematic reviews. J Phys. 2018;64(2):133. https://doi.org/10.1016/j.jphys. 2018.02.015.

14. Sherrington C, Herbert RD, Maher CG, Moseley AM. PEDro. A database of randomized trials and systematic reviews in physiotherapy. Man Ther. 2000;5(4):223-6. https://doi.org/10.1054/math. 2000.0372.

15. Osborne LA, Whittall CM, Emanuel R, Emery S, MD DPPR. Randomized controlled trial of the effect of a brief telephone support intervention on initial attendance at physiotherapy group sessions for pelvic floor problems. Arch Phys Med Rehabil. 2017;98: 2247-52. https://doi.org/10.1016/j.apmr.2017.03.033.

16. Carrión Pérez F, Rodríguez Moreno MS, Carnerero Córdoba L, Romero Garrido MC, Quintana Tirado L, García Montes I. Tratamiento de la incontinencia urinaria de esfuerzo mediante telerrehabilitación. Estudio piloto [Telerehabilitation to treat stress urinary incontinence Pilot study]. Med Clin (Barc). 2015;144(10): 445-8. https://doi.org/10.1016/j.medcli.2014.05.036.

17. Kristjánsdóttir $\mathrm{OB}$, Fors EA, Eide E, et al. A smartphone-based intervention with diaries and therapist-feedback to reduce catastrophizing and increase functioning in women with chronic widespread pain: randomized controlled trial. J Med Internet Res. 2013;15(1):e5. https://doi.org/10.2196/jmir.2249.

18. Kristjánsdóttir ÓB, Fors EA, Eide E, et al. A smartphone-based intervention with diaries and therapist feedback to reduce catastrophizing and increase functioning in women with chronic widespread pain. Part 2: 11-month follow-up results of a randomized trial. J Med Internet Res. 2013;15(3):e72. https://doi.org/10. 2196/jmir.2442. 
19. Sjöström M, Umefjord G, Stenlund H, Carlbring P, Andersson G, Samuelsson E. Internet-based treatment of stress urinary incontinence: a randomised controlled study with focus on pelvic floor muscle training. BJU Int. 2013;112(3):362-72. https://doi.org/10. 1111/j.1464-410X.2012.11713.x.

20. Sjostrom M, Umefjord G, Stenlund H, Carlbring P, Andersson G, Samuelsson E. Internet-based treatment of stress urinary incontinence: 1- and 2-year results of a randomized controlled trial with a focus on pelvic floor muscle training. BJU Int. 2015;116:955-64. https://doi.org/10.1111/bju.13091.

21. Sjostrom M, Umefjord G, Lindholm L, Samuelsson E. Costeffectiveness of an internet-based treatment program for stress urinary incontinence. Neurourol Urodyn. 2015;34:244-50. https://doi. org/10.1002/nau.22540.

22. Hui E, Lee PSC, Woo J. Management of urinary incontinence in older women using videoconferencing versus conventional management: a randomized controlled trial. J Telemed Telecare. 2006;12:343-7. https://doi.org/10.1258/135763306778682413.

23. Laver KE, Adey-Wakeling Z, Crotty M, Lannin NA, George S, Sherrington C. Telerehabilitation services for stroke. Cochrane Database Syst Rev. 2020. https://doi.org/10.1002/14651858. CD010255.pub3.

24. Russell TG. Telerehabilitation: a coming of age. Aust J Physiother. 2009;55(1):5-6. https://doi.org/10.1016/s0004-9514(09)70054-6.
25. Glavind K, Laursen B, Jaquet A. Efficacy of biofeedback in the treatment of urinary stress incontinence. Int Urogynecol J Pelvic Floor Dysfunct. 1998;9(3):151-3. https://doi.org/10.1007/ bf02001084.

26. Mørkved S, Bø K, Fjørtoft T. Effect of adding biofeedback to pelvic floor muscle training to treat urodynamic stress incontinece. Obstet Gynecol. 2002;100(4):730-9. https://doi.org/10.1016/s00297844(02)02160-9.

27. Tiffin N, George A, LeFevre AE. How to use relevant data for maximal benefit with minimal risk: digital health data governance to protect vulnerable populations in low-income and middleincome countries. BMJ Glob Health. 2019;114(2):e001395. https://doi.org/10.1136/bmjgh-2019-001395.

28. Holland AE. Telephysiotherapy: time to get online. Aust J Phys. 2017 Oct;63(4):193-5. https://doi.org/10.1016/j.jphys.2017.08. 001.

29. Ferreira CHJ, Driusso P, Haddad JM, Pereira SB, Fernandes ACNL, Porto D, et al. A guide to physiotherapy in urogynecology for patient care during the COVID-19 pandemic. Int Urogynecol J. 2020;28:1-8. https://doi.org/10.1007/s00192-020-04542-8.

Publisher's note Springer Nature remains neutral with regard to jurisdictional claims in published maps and institutional affiliations. 